rest 3 mothers did not use any supplementation nor any other drugs. One baby had also anencephaly and was lost on the 2nd day of life. The rest babies had hydrocephalus, corpus callosum agenesis, meningomyelocele and underwent surgery. One baby has serious feeding problems thus is on nasogastric feeding. The surviving babies are between 10-26 months, all having physical rehabilitation. Physically the 4 babies are below 3rd percentile both for weight and height. In conclusion, occipital encephalocele is a life-threatening cranial anomaly. The overall outcome of the patient depends on the site and dimension of the lesion, as well as presence of accompanying congenital anomalies. Close multidisciplinary follow-up is needed. FA supplemantation should be nationally provoked.

\section{SEVEN DAY ANHEPATIC SURVIVAL IN A 19 MONTH OLD CHILD, AN INTERDISCIPLINARY CHALLENGE}

doi:10.1136/archdischild-2012-302724.0578

${ }^{1} \mathrm{H}$ Ringe, 'V Varnholt, ${ }^{2} \mathrm{~W}$ Luck, ${ }^{3} \mathrm{G}$ Puhl. ${ }^{1}$ Paediatric Intensive Care; ${ }^{2}$ Paediatric Gastroentrology; ${ }^{3}$ Department of General, Visceral, and Transplantation Surgery, Charite Campus Virchow, Berlin, Germany

Objective Description of pediatric intensive care and surgical management in a 19 month old child after primary liver-graftnon-function, who was managed anhepatic for 8 days in total and re-transplanted twice.

Case Report A 19 month old boy, $10 \mathrm{~kg}$ bodyweight, with ALF of unknown origin received an adult left liver lobe. After all vessels were connected and re-opened the graft showed a massive swelling and perfusion failure due to fulminant micro-vascular rejection and was removed immediately. The portal vein was attached end-toside to the cava inferior. Thereafter diffuse intra-abdominal bleeding occurred, requiring PPSB, factor VII, mass-transfusion and tranexamic-acid and the child was admitted to PICU sedated and ventilated.

To maintain ammonium, bile acids, bilirubin, and cerebral perfusion within thresholds continuous-single-pass-albumin-dialysis (SPAD) on turnover rates up to $150 \mathrm{ml} / \mathrm{kg} / \mathrm{h}$ of hemodiafiltration/filtration was used in total to bridge the anhepatic boy to his first (7 days) and second re-transplantation (1 day). Fresh-frozen-plasma to avoid hemorrhage, water-soluble vitamins, and amino-acids were continuously replaced.

Overall 16 surgical interventions (increased intra-abdominal pressure, portal vein kinking, portal and arterial thrombosis (second graft), removal of mesenterial lymphoid cysts, bile-duct-leak, second re-transplantation with cavo-portal anastomosis, and secondary abdominal wall closure with dermal-porcine-collagen, skinmesh-grafts) and anticoagulation with argatroban were needed to save the boy.

During the 6 month total hospital stay, including 6 weeks on mechanical ventilation, multiple bacterial, viral and fungal infections were detected that required early and timely antimicrobiological treatment.

At 1.5 year follow up the child was alive with intact graft and showed no neurologic sequelae.

\section{CENTRAL VENOUS CATHETER COLONISATION AND CATHETER RELATED SEPSIS: LESSONS LEARNT FROM EXIT SITE SKIN SWAB}

doi:10.1136/archdischild-2012-302724.0579

IV Ponnusamy, ${ }^{2} \mathrm{~V}$ Venkatesh, ${ }^{2} \mathrm{~A}$ Curley, ${ }^{3} \mathrm{~A}$ Perperoglou, ${ }^{4} \mathrm{~N}$ Brown, ${ }^{5} \mathrm{C}$ Tremlett, 'P Clarke. 'Neonatal Intensive Care Unit, Norfolk and Norwich University Hospitals NHS Foundation Trust, Norwich; ${ }^{2}$ Neonatal Intensive Care Unit, Cambridge University Hospitals NHS Foundation Trust, Cambridge; ${ }^{3}$ Norwich Medical School, University of East Anglia, Norwich; ${ }^{4}$ Clinical Microbiology, Cambridge University Hospitals NHS Foundation Trust, Cambridge; ${ }^{5}$ Department of Microbiology, Norfolk and Norwich University Hospitals NHS Foundation Trust, Norwich, UK
Background and Aim Percutaneous central venous catheters (PCVCs) are commonly inserted in neonates after topical antisepsis Presence of a PCVC is a risk factor for catheter-related sepsis (CRS). We examined the relationship between bacteriology of exit site skin swabs (ESSS) taken at line removal and line colonisation/CRS.

Design/methods For all PCVCs removed, ESSS and three separate PCVC segments (proximal, middle and tip) were sent for bacteriological culture. For clinically-septic neonates a peripheral blood culture was additionally obtained. PCVC colonisation was defined as a positive growth in any PCVC segment from a well neonate. Definite CRS was defined as positive growths with the same organism in any PCVC segment plus the blood culture from a clinically-septic neonate.

Results ESSS were culture-positive for 39/187 (21\%) lines removed. Univariate analysis showed that with a positive ESSS, line colonisation was 8 times higher (log odds ratio 2.13 [95\%CI: 1.18-3.08], $\mathrm{p}<0.001$ ), and definite CRS was 14 times higher (2.63 [1.14-4.14], $\mathrm{p}<0.005)$. Adjusting for various covariates, multivariate analysis using a logistic regression model confirmed an increased risk of CRS with a positive ESSS (log odds ratio 2.00 [95\%CI: 0.44-3.58], p=0.01). Conclusion Positive ESSS correlate strongly with PCVCcolonisation and definite CRS. Improved topical antisepsis, skin and catheter care is required to reduce the risk of colonised skin insertion sites associated with catheter placement, and the consequent risks of line colonisation and subsequent CRS.

\section{A SYSTEMATIC APPROACH TO PREVENTING CENTRAL VENOUS CATHETER-ASSOCIATED BLOODSTREAM INFECTION IN PATIENTS RECEIVING HOME PARENTERAL NUTRITION}

doi:10.1136/archdischild-2012-302724.0580

$1 \mathrm{~J}$ Gray, ${ }^{2} \mathrm{C}$ Holden, ${ }^{2} \mathrm{E}$ Sexton, ${ }^{3} \mathrm{~S}$ Pearce. ${ }^{1}$ Department of Microbiologyl; ${ }^{2}$ Nutritional Care; Infection Control, Birmingham Children's Hospital NHS Foundation Trust, Birmingham, UK

Background and Aims Central venous catheter-associated bloodstream infection (CBSI) is a serious complication in home parenteral nutrition (HPN) patients. Prevention of CBSI in hospitals is well-established, but in the home environment presents additional challenges. We applied hazard analysis and critical control points principles to develop a systematic approach to preventing HPNrelated CBSI. Here we describe the corrective actions and their clinical impact.

Methods Factors predisposing to infection, and corrective actions were identified through consensus by a multidisciplinary group of healthcare professionals between April and June 2012. The impact of these actions on CBSI was observed.

Results Key corrective actions were:

1. Staphylococcus aureus nasal screening and decolonisation where appropriate.

2. Multidisciplinary discharge planning meetings included consideration of patients' microbiology histories and optimisation of treatment of comorbidities (e.g. atopic eczema).

3. Increased monitoring of line access and care practices at home, aiming to attain the same standards as in hospital.

4. Launch of updated illustrated guide to identification and management of line site problems.

5. Streamlining of microbiology result reporting to facilitate early treatment of local infections.

6. Taurolidine locks for one patient with a poor line infection history.

Between April 2011 and March 2012 there were 13 CBSI acquired outside hospital, and during planning and implementation of corrective actions (April-June 2012) there were 4 CBSI. By contrast there was only one CBSI during the in the nine months following full implementation. 
Conclusion A multidisciplinary approach to the ongoing management of HPN patients can dramatically reduce their risk of CBSI

\section{PROSPECTIVE STUDY OF CENTRAL VENOUS CATHETER INFECTIONS IN CRITICALLY ILL CHILDREN AT A REGIONAL PAEDIATRIC INTENSIVE CARE UNIT}

doi:10.1136/archdischild-2012-302724.0581

H Gowda, C Penrose. Paediatric Intensive Care Unit, Leeds Teaching Hospitals NHS Trust, Leeds, UK

Background and Aims Central venous catheters (CVC) play an important role in the management of critically ill children, serving both as a reliable vascular access and site of venous pressure monitoring.

To find the incidence CVC related infection and risk factors associated with these complications from different central venous access sites in critically ill children.

Methods A prospective study (August 2010 to July 2011) of 227 central venous catheterisations in children of different ages in a large regional Paediatric Intensive Care Unit. Data on demography, site of insertion and type of CVC infections were collected.

Results Total number of CVC days (for 227 CVC) was 960 days.

Abstract 581 Table 1 CVC infections

\begin{tabular}{lcccc}
\hline $\begin{array}{l}\text { Category of CVC } \\
\text { infection }\end{array}$ & $\begin{array}{c}\text { Catheter related Clinical suspicion of } \\
\text { bacteremia }\end{array}$ & $\begin{array}{c}\text { Local } \\
\text { catheter infection }\end{array}$ & $\begin{array}{c}\text { Catheter } \\
\text { infection }\end{array}$ \\
\hline colonisation \\
\hline pumber of cases & 7 & 14 & 1 & 11 \\
\hline
\end{tabular}

Abstract 581 Table 2 Catheter related bacteremia

\begin{tabular}{lc}
\hline Type of bacteria & Number of cases \\
\hline Coagulase negative staphylococcus & $4 / 7$ \\
Staphylococcus aureus & $2 / 7$ \\
Escherichia coli & $1 / 7$ \\
\hline
\end{tabular}

Abstract 581 Table 3 Internal jugular vein vs femoral vein

\begin{tabular}{|c|c|c|}
\hline & Internal jugular vein & Femoral vein \\
\hline CVC infection & $11 / 127(8.66 \%)$ & $11 / 90(12.22 \%)$ \\
\hline Catheter related bacteremia & $3 / 11$ & $4 / 11$ \\
\hline
\end{tabular}

Relative risk (RR) for femoral vein vs internal jugular vein for CVC infections is 1.4 .

Conclusion Incidence of catheter related bacteremia was 7.29 per 1000 catheter days. Femoral lines have marginal higher infections compared to internal jugular lines.

\section{RISK FACTORS FOR CENTRAL-LINE ASSOCIATED BLOODSTREAM INFECTIONS IN CRITICALLY ILL NEONATES}

doi:10.1136/archdischild-2012-302724.0582

M Pavcnik-Arnol, G Kalan. Department of Pediatric Surgery and Intensive Care, University Medical Centre Ljubljana, Ljubljana, Slovenia

Background and Aims The improvement of central line insertion and maintenance practices has led to reduction but not elimination of central line-associated bloodstream infections (CLABSIs) in critically ill neonates. The objective of our study was to describe CLABSI epidemiology in critically ill neonates in order to identify risk factors for additional prevention strategies.

Methods Prospective cohort study in medical-surgical neonatal and pediatric ICU. All neonates admitted between January 1, 2011, and January 31, 2012 with $\geq 1$ central lines were monitored for the development of CLABSI. Risk factors for CLABSI were examined.

Results Nine CLABSIs occurred during 1640 central line-days (5.5/1000 central line-days). Seven out of 9 CLABSIs (78\%) occurred in neonates with primary surgical diagnosis (gastrointestinal tract anomaly 4, congenital heart disease 2 , rabdomyosarcoma 1), two CLABSIs developed in neonates with hypoxic-ischemic encephalopathy. Neonates with CLABSI had significantly longer ICU length of stay compared to other neonates requiring central line (median 27 vs. 3 days; $p<0.0001$ ), higher PRISM III score (median 14.5 vs. 7; $\mathrm{p}=0.01$ ), more surgical procedures (median 2 vs. $1 ; \mathrm{p}=0.14$ ), longer duration of parenteral nutrition (median 10 vs. 0 days; $p<0.0001$ ), received more red blood cell transfusions (median 5 vs. $0 ; p<0.0001$ ), needed more vasopressor/inotropic agents (median 2 vs. $0 ; p=0.002$ ). Conclusion The incidence of CLABSI is significantly higher in neonates with primary surgical diagnosis (particularly gastrointestinal tract anomaly) and with hypoxic-ischemic encephalopathy compared to other neonates requiring a central line. The increased risk of CLABSI in these neonates warrants further study for development of additional prevention strategies.

\section{THREE YEAR SURVEY OF BLOOD STREAM INFECTIONS (BSI) IN A PAEDIATRIC INTENSIVE CARE UNIT (PICU)}

doi:10.1136/archdischild-2012-302724.0583

'A Gupta, ${ }^{1} \mathrm{~A}$ Top, ${ }^{2} \mathrm{M}$ Farrington. ${ }^{1}$ Paediatric Intensive Care Unit; ${ }^{2}$ Microbiology, Addenbrookes Hospital, Cambridge, UK

Background and Aims Nosocomial infection is a common cause of morbidity and mortality in PICU. Timely microbiological surveillance and assessment of antimicrobial resistance is important in treatment. We studied the microbiological spectrum and susceptibility pattern of pathogens in PICU over 3 years.

Methods Results of blood cultures and antimicrobial susceptibility were reviewed retrospectively over a three year period (April 2008 to 2011). The incidence of nosocomial infections and associated pathogens was analyzed. Positive blood cultures after 48hours of admission were considered as nosocomial BSI.

Results There were 1653 PICU admissions of which 836 patients stayed for more than 48 hours. Overall incidence of BSI was 3.6\% (60 episodes in 1653 patients). Incidence of nosocomial BSI was $2.9 \%$ (24 episodes in 836 patients).

During first 48 hours there were 36 positive blood cultures, 24 Gram-positive (67\%) and 12 Gram-negative (33\%). After 48 hours, there were 24 positive cultures, 20 Gram-positive (83\%) and 4 Gram-negative (17\%). Coagulase negative staphylococcus was the commonest isolate (13/24 nosocomial infections). Other nosocomial pathogens were Staphylococcus aureus $(n=4)$, Enterococus ( $\mathrm{n}=3)$, E. coli, Klebsiella, Pseudomonas and Acinetobacter (1 each).

Varying degree of resistance observed to first line antibiotics (penicillin, gentamicin, 3rd generation cephalosporins). No resistance to second and third line antibiotics (vancomycin, meropenem and tazocin).

Conclusions Gram positive organisms are the predominant cause of nosocomial infections in this study. No multiresistant organisms isolated. However, judicious use of antibiotics is important to prevent emergence of multiresistant strains.

\section{CATHETER ASSOCIATED BLOOD STREAM INFECTION IN PEDIATRIC INTENSIVE CARE}

doi:10.1136/archdischild-2012-302724.0584

${ }^{1} \mathrm{E}$ İnce, ${ }^{2} \mathrm{~T}$ Kendirlii, ${ }^{3} \mathrm{~A}$ Yaman, ${ }^{3} \mathrm{C}$ Ödek, ${ }^{4} \mathrm{~A}$ Karbuz, ${ }^{5} \mathrm{~B}$ Aldemir, ${ }^{4} \mathrm{H}$ Özdemir, ${ }^{4} \mathrm{E}$ Çiftçi, ${ }^{6} \mathrm{H}$ Güriz, ${ }^{6} \mathrm{D}$ Aysev. ${ }^{1}$ Pediatric Infectious Disease; ${ }^{2}$ Pediatric ICU, Ankara University, Faculty of Medicine; ${ }^{3}$ Pediatric ICU; ${ }^{4}$ Pediatric Infectious Disease, Ankara University, Ankara; ${ }^{5}$ Pediatric Infectious Disease, Ankara University, Adana; ${ }^{6}$ Microbiology Laboratory, Ankara University, Ankara, Turkey 